\title{
Disease concordance and sex similarity in twins
}

\author{
T. MARSHALL AND E. G. KNOX \\ From the Department of Social Medicine, University of Birmingham
}

INTRODUCTION Disease patterns in twins may show the presence of genetic or of environmental causes. Two arguments of inference have been used. Discordances between monozygotic twins have been taken to indicate the presence of environmental determinants; and high levels of concordance in monozygotic (MZ) pairs compared with dizygotic (DZ) pairs have been taken to indicate the presence of genetic causes. Neither argument is rigorous and, at this level, neither is quantitative.

An investigator cannot always establish the zygosity of individual twin pairs, and many have therefore used indirect arguments, based upon Weinberg's differential method. ${ }^{1}$ Thus, the number of $\mathrm{MZ}$ pairs in a randomly selected sample of twin pairs may be estimated by subtracting the number of unlike-sexed (U) from the number of like-sexed pairs (L). The proportion of $M Z$ pairs is then $(\mathrm{L}-\mathrm{U}) /(\mathrm{L}+\mathrm{U})$, and of $\mathrm{DZ}$ pairs $2 \mathrm{U} /(\mathrm{L}+\mathrm{U})$. Cannings ${ }^{2}$ showed that in twins which are randomly selected, and when the proportion of males is $0 \cdot 5$, these formulae are maximum-likelihood estimators; also that their validity is unaffected by differential prenatal viability of $\mathrm{MZ}$ and $\mathrm{DZ}$ twins.

However, in the context of investigating aetiologies we need to ask whether these relationships remain true in twins which are not selected randomly, but because a particular disease occurred in one or both of the pair. This distinction has not always been clearly made in the past. It turns out to be crucial.

Smith, ${ }^{3}$ for example, was led to an erroneous conclusion with respect to the aetiology of Down's disease. This he later recognised, ${ }^{4}$ and showed that the formulation of a correct method for estimating the proportions of the $\mathrm{MZ}$ and $\mathrm{DZ}$ twins depended upon the prior choice of a model of pathogenesis. It was necessary to decide in advance whether the disease-determining events occurred before or after the point of MZ cleavage. Distortions of the Weinberg rule also occur in sex-linked recessive transmission where the $U: L$ ratio among $D Z$ pairs is other than $1: 1$; it is this ratio upon which the validity of the Weinberg method chiefly depends. For the investigator examining diseases the causes of which are not known, the necessity to make assumptions about the aetiology of the disease before he even begins his analysis introduces a disturbing circularity. These considerations provide the background to this paper.

We tackle the problems described above in three stages. Firstly, we display more formally the limitations both of the Weinberg rule and of concordance estimates, in relation to simple genetic models. Secondly, we devise a general analytical framework which largely circumvents these problems

Authors are reminded that manuscripts must henceforward be submitted in the Vancouver style. ${ }^{12}$ One of the requirements of the new system is that references should be identified in the text by arabic numerals, either superscript or in brackets, and numbered consecutively in the reference list in the order in which they were first mentioned in the text. Journal titles should be abbreviated according to the style of Index Medicus and authors should supply the first and the last page numbers of an article or chapter. The names of all authors should be given if there are fewer than seven; if there are more, give the first three followed by $e t$ al.

${ }^{1}$ International Steering Committee of Medical Editors. Uniform requirements for manuscripts submitted to biomedical journals. Br Med J 1979 ; 1: 532-5.

'Journal of Epidemiology and Community Health. Change of style. J Epidemiol Community Health 1979; 33: 171. 
and allows us to make causal inferences from twin data. Thirdly, we demonstrate the use of the new model in relation to published data.

PART I: SIMPLE GENETIC MODELS

We consider three simple genetic models. In each case, the deleterious gene is rare, and the appropriate genotype is fully penetrant. The models are (1) autosomal recessive transmission with both parents heterozygous, (2) X-borne recessive transmission, where the mother is a carrier, and (3) dominant transmission with one parent heterozygous.

In the general population, the proportion of male fertilised ova is $m(f=1-m)$; the proportion of $M Z$ twin pairs is $p$, and of $D Z$ pairs $q(q=1-p)$. We assume that parental carriage of the deleterious genes is independent of the risk of twinning, or of zygosity, and each is independent of the sex of the zygote.

Our purpose now is to estimate for each model the proportion (A) of affected twin pairs which are in fact $\mathrm{MZ}$, and to relate this both to the value (W) obtained by applying Weinberg's rule, as well as to the proportion ( $p$ ) in the population at large.

The process is illustrated in Table 1, where A and $\mathrm{W}$ are derived for each of the models, and for a general genetic model which covers all of them. The general model uses two values, namely $\mathrm{g}$ (the risk in boys) and $h$ (the risk in girls) which may be substituted (for example, $1 / 4: 1 / 4 ; \frac{1}{2}: 0 ; 1 / 2: 1 / 2$ in the three special models). For the two autosomal models discrepancies between $A$ and $W$ depend on the term $(m-f)^{2}$; and where $m=f, A$ and $W$ are identical. However, this is not true in the case of sex-linked recessive transmission, where $\mathrm{A}$ and $\mathrm{W}$ are widely different and where, for low values of $p$, Weinberg's formula can give negative proportions.

Where $\mathrm{g}=\mathrm{h}=1 \cdot 0, \mathrm{~W}$ reduces to $\mathrm{p}+\mathrm{q}(\mathrm{m}-\mathrm{f})^{2}$; and when in addition $\mathrm{m}=\mathrm{f}=1 / 2$, then $\mathrm{W}=\mathrm{p}$. This represents the case where all twins in the population are selected. In all other cases $p$ is different both from $A$ and from $\mathrm{W}$. That is, the MZ proportion in the general population is never the same as among twin pairs affected by genetically determined diseases, and cannot be used as a guide to the 'representativeness' of aggregates of such pairs.

\section{Relationship with concordance estimates}

There are two measures of concordance in common use (see Allen et al. ${ }^{5}$ for a full discussion). The Pairwise Concordance Rate is the proportion of concordant twin pairs among all affected twin pairs;

Table 1 Distribution of affected twin pairs by zygosity and sex type for simple genetic models

\begin{tabular}{|c|c|c|c|c|c|c|c|c|}
\hline & \multicolumn{2}{|c|}{ Autosomal recessive } & \multicolumn{2}{|c|}{$X$-borne recessive } & \multicolumn{2}{|c|}{ Autosomal dominant } & \multicolumn{2}{|c|}{ General genetic model } \\
\hline & $M Z$ & $D Z$ & $M Z$ & $D Z$ & $M Z$ & $D Z$ & $M Z$ & $D Z$ \\
\hline$\overline{\mathbf{M}} \overline{\mathbf{M}}$ & $\frac{1}{4} \mathrm{mp}$ & $\frac{1}{16} m^{2} q$ & $\frac{1}{2} \mathrm{mp}$ & $\frac{1}{4} m^{2} q$ & $\frac{1}{2} \mathrm{mp}$ & $\frac{1}{4} m^{2} q$ & $g \cdot m p$ & $g^{2} \cdot m^{2} q$ \\
\hline$\overline{\mathbf{M}} \mathbf{M}$ & - & $\frac{6}{16} m^{2} q$ & - & $\frac{1}{2} m^{2} q$ & - & $\frac{1}{2} m^{2} q$ & - & $2 g(1-g) \cdot m^{2} q$ \\
\hline$\overline{\mathbf{F}} \overline{\mathbf{F}}$ & $\frac{1}{4} \mathrm{fp}$ & $\frac{1}{16} f^{2} q$ & - & - & $\frac{1}{2} \mathrm{fp}$ & $\frac{1}{4} \mathrm{f}^{2} \mathrm{q}$ & $h \cdot f p$ & $h^{2} \cdot f^{2} q$ \\
\hline$\overline{\mathrm{F} F}$ & - & $\frac{6}{16} f^{2} q$ & - & - & - & $\frac{1}{2} f^{2} q$ & - & $2 h(1-h) \cdot f^{2} q$ \\
\hline$\overline{\mathbf{M}} \overline{\mathbf{F}}$ & - & $\frac{2}{16} \mathrm{mfq}$ & - & - & - & $\frac{1}{2} \mathrm{mfq}$ & - & $\mathrm{gh} \cdot 2 \mathrm{mfq}$ \\
\hline$\overline{\mathbf{M}} \mathbf{F}$ & - & $\frac{6}{16} \mathrm{mfq}$ & - & $\mathrm{mfq}$ & - & $\frac{1}{2} \mathrm{mfq}$ & - & $g(1-h) \cdot 2 m f q$ \\
\hline $\mathbf{M} \overline{\mathbf{F}}$ & - & $\frac{6}{16} \mathrm{mfq}$ & - & - & - & $\frac{1}{2} \mathrm{mfq}$ & - & $(1-g) h \cdot 2 m f q$ \\
\hline $\mathbf{A}$ & $\overline{4}$ & & $2 \overline{p+}$ & $\sqrt{4 f})$ & & & $\frac{M}{M Z L+D}$ & $\overline{\mathrm{DZU}}$ \\
\hline $\mathbf{w}$ & $\frac{4 p+}{4}$ & $\frac{-f)^{2}}{1}$ & $\frac{2 p+}{2 p+}$ & $\frac{-4 f)}{+4 f)}$ & $\frac{2 p+3}{2 p}$ & $-f^{2}$ & $\frac{M Z L+D}{M Z L+D}$ & $\frac{\mathrm{DZU}}{\mathrm{DZU}}$ \\
\hline
\end{tabular}

Where $M Z L=p(g m+h f)$

$D Z L=q\left(g(2-g) m^{2}+h(2-h) f^{2}\right)$

$D Z U=2 m f q(g+h-g h)$ 
Table 2 Concordance estimates for simple genetic models

\begin{tabular}{|c|c|c|c|c|c|c|c|c|}
\hline & \multicolumn{2}{|l|}{ Autosomal recessive } & \multicolumn{2}{|l|}{$X$-borne recessive } & \multicolumn{2}{|l|}{ Autosomal dominant } & \multicolumn{2}{|c|}{ General genetic model } \\
\hline $\begin{array}{l}\text { CONCORDANCE RATE } \\
\text { (ALGEBRAIC) }\end{array}$ & L & $\mathbf{U}$ & $\mathbf{L}$ & $\mathbf{U}$ & $\mathbf{L}$ & $\mathbf{U}$ & $\mathbf{L}$ & $\mathbf{U}$ \\
\hline Pairwise & $\frac{4 p+q\left(m^{2}+f^{2}\right)}{4 p+7 q\left(m^{2}+f^{2}\right)}$ & $\frac{1}{7}$ & $\frac{2 p+m q}{2 p+3 m q}$ & - & $\frac{2 p+q\left(m^{2}+f^{2}\right)}{2 p+3 q\left(m^{2}+f^{2}\right)}$ & $\frac{1}{3}$ & $\frac{\mathrm{LC}}{\mathrm{LC}+\mathrm{LD}}$ & $\frac{g h}{g+h-g h}$ \\
\hline Proband & $\frac{4 p+q\left(m^{2}+f^{2}\right)}{4 p+4 q\left(m^{2}+f^{2}\right)}$ & $\frac{1}{4}$ & $\frac{2 p+m q}{2 p+2 m q}$ & - & $\frac{2 p+q\left(m^{2}+f^{2}\right)}{2 p+2 q\left(m^{2}+f^{2}\right)}$ & $\frac{1}{2}$ & $\frac{2 L C}{2 L C+L D}$ & $\frac{2 g h}{g+h}$ \\
\hline \multicolumn{9}{|l|}{$\begin{array}{l}\text { CONCORDANCE RATE } \\
\text { (ARITHMETIC) }{ }^{\dagger}\end{array}$} \\
\hline Pairwise & 0.514 & $0 \cdot 143$ & 0.647 & - & 0.647 & 0.333 & \multirow{2}{*}{\multicolumn{2}{|c|}{$\begin{array}{l}\text { Indeterminate without prior choice } \\
\text { of model and/or prior knowledge } \\
\text { of } m \text { and } p\end{array}$}} \\
\hline Proband & 0.679 & $0 \cdot 250$ & 0.786 & - & 0.786 & 0.5 & & \\
\hline
\end{tabular}

Where $L C=p(g m+h f)+q\left(g^{2} m^{2}+h^{2} f^{2}\right)$ $L D=2 q\left[g(1-g) m^{2}+h(1-h) f^{2}\right]$

† For $m=f=0.5 ; p=0.4$

that is, $C /(C+D)$ where $C$ is the number of concordant, and $\mathrm{D}$ is the number of discordant, pairs. The Proband Concordance Rate is the proportion of affected co-twins, when a first twin is known to be affected; that is, $2 \mathrm{C} /(2 \mathrm{C}+\mathrm{D})$.

Table 2 provides concordance expressions for the models described in Table 1. The rates are expressed algebraically in the upper part of the table and numerically, following substitution, in the lower part. In both parts, the values are presented separately for like-sexed and unlike-sexed pairs. Concordance estimates for U-pairs appear as simple proportions, except in the case of the sex-linked model, when the value is indeterminate. The values for $L$-pairs are all indeterminate without reference to external data; these include the values $p$ and $\mathrm{m}$. More importantly, evaluation depends upon the choice of model which, circularly, is the point in question.

Substitution in the formulae given in Table 2 shows that (except when $g=h=1$ ) concordance rates are always greater in L-pairs than in U-pairs. It is this which invites the qualitative genetic interpretations described in our first paragraph, when such differences are found. However, the extent of the difference depends as much upon the particular choice of genetic model as it does upon the penetrance of the genotype, and without such prior choice there is no way in which the difference can be used to indicate the strength of the genetic component. There is no way in which such differences can be used as quantitative indicators of the strength of a hypothetical genetic component when the aetiology is complex or undefined.

PART II: GENERAL ANALYTIC MODEL

The models described above assume full penetrance. That is, there are no necessary environmental causes. For aetiologies containing environmental components, the outcomes will depend both upon their strength and upon their timing. Smith ${ }^{3} 4$ pointed out that disease-determining events which strike before the point of cleavage will produce different proportions of affected $\mathrm{MZ}$ and $\mathrm{DZ}$ pairs, compared with similar events occurring after this point in time. Events affecting zygotes (independently) before the point of cleavage will select $\mathrm{MZ}$ and $\mathrm{DZ}$ pairs from the parent population with relative frequencies of $1: 2$, while similar risks applied after cleavage will result in relative frequencies of $1: 1$. Our task now is to construct a model which will accommodate both kinds of event operating alone or in combination.

Table 3 displays the distribution of a disease the incidence of which in a susceptible subset of the population (as above) is $i$. The different sex and concordance types are presented separately for $\mathrm{MZ}$ and $\mathrm{DZ}$ pairs. The DZ calculations assume independent risks to the two zygotes. For $M Z$ pairs two alternative solutions are available and both are presented; the first corresponds with a risk to a

Table 3 Relative distributions of affected $M Z$ and $D Z$ pairs

\begin{tabular}{|c|c|c|c|}
\hline & \multicolumn{2}{|l|}{$M Z$} & \multirow[b]{2}{*}{$D Z$} \\
\hline & Pre-cleavage & Post-cleavage & \\
\hline$\overline{\mathbf{M}} \overline{\mathbf{M}}$ & pmi & $\mathrm{pmi}^{2}$ & $q m^{2} i^{2}$ \\
\hline$\underline{\overline{\mathbf{M}}} \mathbf{M}$ & - & $2 \operatorname{pmi}(1-i)$ & $2 q m^{2} i(1-i)$ \\
\hline$\overline{\mathbf{F}} \overline{\mathbf{F}}$ & pfi & $\mathrm{pfi}^{2}$ & $q^{2} i^{2}$ \\
\hline$\overline{\mathbf{F} F}$ & - & $2 \operatorname{pfi}(1-i)$ & $2 q f^{2} i(1-i)$ \\
\hline$\overline{\mathbf{M}} \overrightarrow{\mathbf{F}}$ & - & - & $2 q \mathrm{mf}^{2}$ \\
\hline$\overline{\mathbf{M}} \mathbf{F}$ & - & - & $2 q m f i(1-i)$ \\
\hline$M \bar{F}$ & - & - & $2 q m f i(1-i)$ \\
\hline
\end{tabular}

MF represent affected twins

$\mathrm{i}=$ incidence of disease independent of sex and zygosity 
pre-cleavage zygote, and the second corresponds with independent risks to each of the post-cleavage embryos. This follows Smith's formulation. Row totals cannot be calculated in this table unless a choice is first made between the two MZ models.

Table 4 develops the algebra of Table 3 through two further stages. Firstly, it allows different incidences in males and females; and secondly, it resolves each estimate of incidence into two elements ( $a$ and $b$ in males, $c$ and $d$ in females) representing the probabilities of two events. Both events are necessary to cause the disease, and the incidence in the stratum-at-risk is equivalent to the product of their (supposedly) independent probabilities. The two events are differentiated with respect to the point of cleavage in $\mathrm{MZ}$ twins, and the equivalent time in $\mathrm{DZ}$ twins; $a$ and $c$ are pre-cleavage risks, and $b$ and $d$ are post-cleavage risks. This allows the combination of the first two columns in Table 3 into a single column, and permits designation of row totals in Table 4 .

The seven algebraic expressions can be set against observed relative frequencies of twin pairs, leading in principle to a solution of the four unknown values a, b, c, d. Each of the four events represented by these frequencies might eventually be resolved into multiple sub-events, with frequencies linked under appropriate combinatorial rules to reconstitute the composite values. This, however, is beyond our present purposes and we shall limit ourselves to the resolution represented in Table 4 .

In circumstances where it is unnecessary to invoke a pre-cleavage or a post-cleavage event at all, the appropriate 'risk' is unity rather than zero. This value is required by the supposition of necessity, and the fact that the disease occurred. That is, in considering the genetic determinants of otitis media, it is only necessary to have ears; and everyone has ears, so $\mathrm{a}=\mathrm{c}=1 \cdot 0$.
The model laid out in Table 4 successfully reduces to the simple genetic models of Tables 1 and 2 through appropriate substitution. Thus, for fully penetrant autosomal recessive transmission, $a=c=1 / 4$ and $b=d=1 \cdot 0$. For $X$-borne recessive disease $a=\frac{1}{2}: c=0 \cdot 0: b=d=1 \cdot 0$. For all twins in the population $a=b=c=d=1 \cdot 0$. For models of environmentally determined diseases occurring equally in both sexes (otitis media, lightning strike) $\mathrm{a}=\mathrm{c}=1.0$ while $\mathrm{b}=\mathrm{d}=\mathrm{i}$.

The model is appropriate either to situations in which the general population can be regarded as uniformly at risk, or sharply stratified into an at-risk and a not-at-risk layer. The 'incidence' of the disease $(\mathrm{i}=\mathrm{ab}=\mathrm{cd})$ relates to the stratum-at-risk rather than, necessarily, to the whole population. In rhesus haemolytic disease, for example, the at-risk stratum consists of children of D-sensitised mothers with either D-homozygous (about $80 \%$ ) or D-heterozygous husbands. Under these conditions the effect is fully penetrant. Thus, $a=c=0.9$ (approximately) and $b=d=1 \cdot 0$. In many situations values for i obtained from twin data can be compared with incidence estimates obtained from the total population; this will permit an investigator to determine the degree of stratification, and the concentration of risk, within the population at large. Disease distributions exhibiting complex or continuous stratification of risk may defeat the utility of the algebra so far developed, as may certain secondary selective processes such as differential survival. We shall return to these points later.

\section{Solving the parameters}

The equations in Table 4 contain six basic parameters, namely $\mathrm{m}, \mathrm{p}, \mathrm{a}, \mathrm{b}, \mathrm{c}$, and $\mathrm{d}$, together with the complements of $m$ and $p$. However, $p$ is an externally derived value which can usually be

Table 4 Relative distributions of $M Z$ and $D Z$ pairs according to sex differentiation of risk, and time variation of causal events

\begin{tabular}{|c|c|c|c|c|}
\hline & $M Z$ & $D Z$ & Total & \\
\hline$\overline{\mathbf{M}} \overline{\mathbf{M}}$ & $\mathrm{mpab}^{2}$ & $m^{2} q a^{2} b^{2}$ & $m a b^{2}(p+m q a)$ & $t$ \\
\hline$\overline{\mathbf{M}} \mathbf{M}$ & $2 m p a b(1-b)$ & $2 m^{2} q a b(1-a b)$ & $2 m a b[p(1-b)+m q(1-a b)]$ & $\mathbf{u}$ \\
\hline$\overline{\mathbf{F}} \overline{\mathbf{F}}$ & $\mathrm{fpcd}^{2}$ & $f^{2} q c^{2} d^{2}$ & $f c d^{2}(p+f q c)$ & $\mathbf{v}$ \\
\hline$\overline{\mathbf{F}} \mathbf{F}$ & $2 f \operatorname{fpd}(1-d)$ & $2 f^{2} q c d(1-c d)$ & $2 f c d[p(1-d)+f q(1-c d)]$ & $\mathbf{w}$ \\
\hline$\overline{\mathbf{M}} \overline{\mathbf{F}}$ & - & $2 \mathrm{mfqabcd}$ & $2 \mathrm{mfqabcd}$ & $\mathbf{x}$ \\
\hline $\bar{M} F$ & - & $2 \mathrm{mfqab}(1-\mathrm{cd})$ & $2 m f q a b(1-c d)$ & $\mathbf{y}$ \\
\hline$M \bar{F}$ & - & $2 \operatorname{mfqcd}(1-a b)$ & $2 m f q c d(1-a b)$ & $\mathbf{z}$ \\
\hline
\end{tabular}

$a=$ incidence of events in males pre-cleavage or equivalent time in dizygotic twins.

$b=$ incidence of events in males post-cleavage or equivalent time in dizygotic twins.

$c=$ incidence of events in females pre-cleavage or equivalent time in dizygotic twins.

$d=$ incidence of events in females post-cleavage or equivalent time in dizygotic twins. 
measured accurately in the parent population, and we should usually opt for substitution rather than solution. Solving for $\mathrm{m}$ is equally inappropriate; in most populations it is sufficiently close to 0.5 . In addition, a disease with an asymmetric sex ratio may be said arbitrarily to occur in a stratum with (i) equal numbers of males and females displaying different incidences, or (ii) unequal subpopulations of males and females with identical incidences. Any inaccuracies in setting $\mathrm{m}=\mathrm{f}=0.5$ will be automatically compensated for in an appropriate solution for the four risk-parameters a,b,c, and d.

The four parameters are over-determined in the seven equations, so direct algebraic solution is not appropriate. Nor does it deal adequately with sampling variation. The derivation of maximum likelihood estimators is possibly the ideal approach. It was attempted but had to be abandoned because of algebraic complexity. In the end, and despite the fact that in some situations it is known to be suspect, ${ }^{6}$ we opted for minimisation of $\chi^{2}$. In this process initial values for each of the parameters are set, the expected distribution of twin pair types is calculated and compared with the observations, and the parameter values adjusted iteratively until a best-fit is obtained between the predictions and the observations. $\chi^{2}$ is used as the measure of goodness of fit.

A particular advantage is that the method serves not only to determine what are the best estimates of $a, b, c$ and d, but also to test whether the model, with these best-fit values, is itself a credible interpretation of the particular situation. With seven values $(\mathrm{t}, \mathrm{u}, \ldots \ldots \mathrm{z})$ and with four determining parameters $(a, b, c, d), \chi^{2}$ is most appropriately tested against two degrees of freedom.

Pragmatically, when applied to simple deterministic models (as earlier), unperturbed by sampling variation, the method produced the right answers.

\section{PART III: APPLICATIONS}

In this section we explore the problems of applying the model described in Part II to real data sets. This exercise is based upon published material relating to five diseases: (a) mongolism (b) schizophrenia (c) coronary occlusion (d) congenital neural tube defects and (e) infantile hypertrophic pyloric stenosis. The distributions of twin pairs according to sex similarity and concordance are given in Table 5, which shows the parameter values estimated by iterative minimisation of $\chi^{2}$. This also provides a measure of the disagreement between the observed and theoretically expected values.

We proposed earlier that iteration should be carried out on the four basic parameters $(a, b, c, d)$,

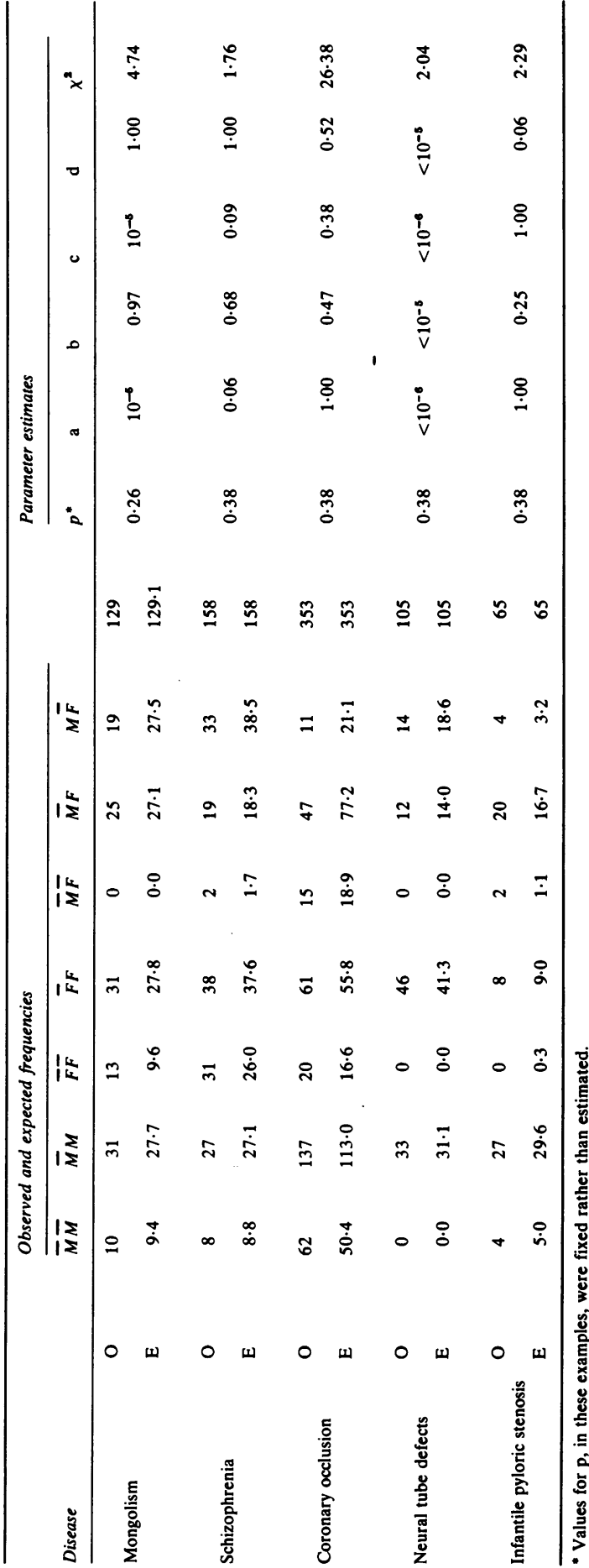

Table 5 Application to published data-sets 
representing the probabilities of pre- and post-cleavage events in males and females, and that a value for $p$, representing the proportion of $M Z$ twins in the general population, should usually be derived from external data. In practice, the appropriate value for $p$ is not always known, so we designed our iterative procedures to operate optionally upon either a fixed or a floating value for $p$.

Our interpretations of the five sets of results are as follows:

\section{(a) Mongolism}

Smith's twin data on mongols, ${ }^{34}$ amounting to 129 pairs, were re-examined using a range of fixed values and floating values for $p$. Probably the most valid was that based upon fixing $p$ at $0 \cdot 263$, a value suggested by Smith and calculated to allow for the late age distribution of mothers giving birth to mongol infants. (The value of $p$ for the general population of England and Wales in 1972 is estimated at 0.38).

The solutions for a,b,c,d, are shown in the Table together with model 'expected' values for the different twin pairs; the minimum $\chi^{2}$ is compatible with the postulate that the model is valid in this situation. The low values for a and c create a problem of interpretation because the products $\mathrm{ab}$ and cd are less than the incidence of the disease in the population, a measured value of about 0.001 . However, this probably arises from a fortuitous absence of unlike-sexed concordant pairs among the observations and, if this can be accepted, we can reach a conclusion about this disease which has not been made before. That is, there is no room for substantial stratification of risk in the population at large (for example, beyond that associated with maternal age) and most women must be at hazard. The estimates of $b$ and $d$ suggest that the pre-cleavage determining event is fully penetrant, and this is entirely compatible with our current understanding of the disease.

\section{(b) Schizophrenia}

Slater ${ }^{7}$ provided data on twin pairs in which one or both had schizophrenia. Depending on the definitions used, there are several different ways of assembling this material. The figures in Table 5 give the results of one such analysis, but all gave similar results. As with mongolism and with simple genetic models, these results suggest selection in the pre-cleavage stage followed by high levels of penetrance. However, the values for the pre-cleavage stage are much higher than in the case of mongolism, and presumably higher than the incidence of schizophrenia in the general population. There is therefore a strong suggestion of stratification of risk in the general population. It should be noted that this conclusion is derived not from the twin data alone, but through a contrast with external information; also that there is nothing intrinsic to the twin data themselves to show whether the basis of this (pre-cleavage) heterogeneity is genetically or environmentally determined, or both. The data do not fit the hypotheses of dominant or recessive inheritance transmitted through a single locus, which would give values for a and co approximately 0.5 or $0 \cdot 25$, whatever penetrance was assumed. The data would, however, be compatible with dependence upon the simultaneous homozygous occurrence of recessive genes at two loci with $\mathrm{a}=\mathrm{c}=0.25^{2}=0.0625$.

\section{(c) Coronary occlusion}

Data supplied by Harvald and Hauge ${ }^{8}$ refer to 358 twin pairs in whom one or both had had coronary occlusion. In this case there is a distinctive asymmetric sex ratio. In iteration based upon all five parameters, minimisation of $\chi^{2}$ gave an estimate for $p$ of 0.62 , a value which is difficult to accept. When $p$ was fixed at $\mathbf{0 \cdot 3 8}$, the current figure for England and Wales, the results were as shown in Table 5 and $\chi^{2}$ was well outside acceptable confidence limits. There are clearly difficulties in fitting any hypotheses to these data within the terms of the model. We conclude, therefore, that the model does not represent the true situation. It is possible that the recorded events encompass several different aetiologies, or that the risks of twinning and of disease are non-independent, or that the twins interacted, or that a simple stratification of risk within the population is an inappropriate representation, or that the sample has been subjected to biased selection, or mortality from competing causes.

In pragmatic terms we conclude that the model is inappropriate to the case and that we cannot illuminate the aetiology of the disease from twin data within our present techniques.

\section{(d) Congenital neural tube defect}

The data assembled by Yen and MacMahon of 105 twin pairs with anencephalus were analysed. Although these twins contained no concordant pairs at all, optimal adjustment of the main parameters resulted in a reasonable fit. However, the values for $a, b, c$, and $d$ were all very small indeed and the incidence estimates derived from the products $a b$, and cd, were infinitesimal. This is a much more extreme result than in the case of our analysis of mongols and cannot easily be attributed to sampling variations. Acceptance of the model demands the impossibility that affected pairs are preferentially selected from a stratum with a very low risk indeed 
and we must therefore conclude again that the model is inappropriate to the circumstance. It follows that we must reject simple recessive and dominant inheritance at any level of penetrance, and also highly penetrant mutations or chromosomal abnormalities. We must also reject extensions of simple mechanisms such as that postulated in the analysis of the schizophrenia data; that is, multiple-locus inheritance is rejected too.

It may be noted that this conclusion has previously been reached on other grounds with respect to this disease, ${ }^{1011}$ it being postulated that fetus-fetus interactions play a part in its aetiology.

\section{(e) Infantile hypertrophic pyloric stenosis}

Data supplied by MacMahon and McKeown ${ }^{12}$ contained 65 twin pairs in which one or both was affected with infantile pyloric stenosis. The result of the analysis given in Table 5 is interesting for several reasons.

Firstly, the products ab and cd are much greater than the population incidences in males and females $(0.005$ and 0.001 respectively) and higher even than the incidence in non-twin sibs of propositi with this disease. There is therefore a high level of stratification within the population, with concentrations of risk not only in particular sibships, but also in individual pregnancies within those sibships.

Secondly, the pre-cleavage factors are too high to be compatible with any simple genetic mechanism. That is, we exclude simple autosomal dominant or recessive inheritance with sex-biased penetrance and we exclude sex-linked recessive inheritance. Additionally, the pre-cleavage factors bear no resemblance to the kinds of results we should expect from polygenic inheritance, as proposed by several authors, notably Carter and Evans. ${ }^{13}$ To our knowledge this is the first time that a method has been devised whereby this hypothesis, with its exceedingly versatile predictions, can be successfully tested and rejected.

The basis of the population heterogeneity remains as before a matter of speculation, but the choice is now much more limited. On the one hand we might postulate a pre-cleavage event, analogous with haemolytic disease, in which only a small part of the population suffers a very high risk while the remainder is safe. If there is a genetic explanation, then it probably operates upon the mother rather than upon the child. On the other hand we could postulate an environmental factor, such as a nutritional idiosyncrasy, as the main cause. In this case the values for a and $c$ are 1.0 simply because it is necessary to have a pylorus before it is possible to have pyloric stenosis. Further clarification must depend upon data external to the twin data, which themselves can offer no further comment. However, the characteristic familial concentrations of the disease, particularly in maternal relatives, suggest a mechanism in the first class rather than in the second.

\section{Discussion}

Our examination of the problems of drawing aetiological inferences from mixed MZ/DZ twin data leads to the following main conclusions.

(1) The use of Weinberg's method, and of concordance estimates, are unsatisfactory. Weinberg's approach, as commonly applied, conceals a confusion between the proportion of $\mathrm{MZ}$ pairs in random samples, and in samples selected on the basis of disease. The use of these methods to infer causal mechanisms depends upon prior assumptions concerning the causal mechanisms themselves.

(2) Complex aetiologies, containing both environmental and genetic deteminants, introduce further complications. A model framework was therefore developed in which a direct approach to the genetic/environmental question was abandoned, and which depends instead upon the timing of the determining events, irrespective of their nature. This model permits the investigation of disease data in twins without requiring an over-restrictive set of prior assumptions. Seven equations represent the frequencies of the seven different kinds of twin pairs in which one or both is affected. The frequencies are declared in terms of four parameters representing the frequencies of determining events before and after the point of cleavage of $\mathrm{MZ}$ pairs, in males and females separately.

(3) The seven equations are set against observations and the parameters are solved. Pragmatically, minimisation of $\chi^{2}$ was the only approach which met our needs. It has the added advantage that, in addition to solving the parameter values, it is capable of detecting (some) situations for which the model is inappropriate.

(4) The timing-based model is easy to use. Applications to published data illustrated the various ways in which inferences might be drawn. In one example (mongolism) where the sequence of events is well understood, the method generated an interpretation compatible with what is already known. In another example (anencephalus) its application led down reasoning pathways which have already been derived on other grounds. In another example (coronary disease) the self-rejecting properties of the model were demonstrated. In the case of schizophrenia a new and reasonably plausible model was suggested. In the case of infantile hypertrophic pyloric stenosis, several existing model explanations were excluded. 
Reprints from Professor E. G. Knox, Department of Social Medicine, Health Services Research Centre, The Medical School, Edgbaston, Birmingham B15 2TJ.

\section{References}

${ }^{1}$ Weinberg W. Beiträge zur physiologie und pathologie der mehrlingsgeburten beim menschen. Pfluegers Arch 1902; 88: 346-430.

${ }^{2}$ Cannings C. A discussion of Weinberg's rule on the zygosity of twins. Ann Hum Genet 1969; 32: 403-5.

${ }^{3}$ Smith A. A note on mongolism in twins. Br J Prev Soc Med 1955; 9: 212-3.

${ }^{4}$ Smith A. A further note on mongolism in twins. Br J Prev Soc Med 1960; 14: 47-8.

${ }^{5}$ Allen G, Harvald B, Shields J. Measures of twin concordance. Acta Genet (Basel) 1967; 17: 475-81.
${ }^{6}$ Fisher RA. Statistical Methods for Research Workers. 10th ed. Edinburgh: Oliver and Boyd, 1946.

${ }^{7}$ Slater E. Psychotic and Neurotic Illnesses in Twins. Medical Research Council Special Report Series No. 278. London: Medical Research Council, 1953.

${ }^{8}$ Harvald B, Hauge M. Coronary occlusion in twins. Acta Genet Med Gemellol 1970; 19: 248-50.

${ }^{9}$ Yen S, MacMahon B. Genetics of anencephaly and spina bifida. Lancet 1968; 2: 623-6.

${ }^{10} \mathrm{Knox}$ EG. Twins and neural tube defects. Br J Prev Soc Med 1974; 28: 73-80.

${ }^{11}$ Knox EG. Fetus-fetus interaction. A model aetiology for anencephalus. Dev Med Child Neurol 1970; 12: 167-77.

${ }^{12}$ MacMahon B, McKeown T. Infantile hypertrophic pyloric stenosis. Data on 81 pairs of twins. Acta Genet Med Gemellol 1955; 4: 320-9.

${ }^{13}$ Carter CO, Evans KA. Inheritance of congenital pyloric stenosis. J Med Genet 1969; 6: 233-54. 